\title{
Hint of Lepton Flavor Violation at the LHC
}

\section{Sanjoy Biswas*广}

School of Physics, Korea Institute for Advanced Study

Seoul 130-722, Republic of Korea

E-mail: sanjoyakias.re.kr

The recent results from the $\mathrm{LHCb}$ in the context of $B^{+} \rightarrow K^{+} \ell \ell$ decay, the CMS analysis in the context of right handed W-boson $\left(W_{R}\right)$ search and CMS searches for the di-leptoquark production show significant deviations from the Standard Model expectations. In this work, we address these seemingly uncorrelated results in the context of $\mathscr{R}$-parity violating supersymmetry. We found that a particular combination of $L Q D^{c}$-type operators which successfully explain the LHCb result, can also accommodate the CMS excesses.

The European Physical Society Conference on High Energy Physics

22-29 July 2015

Vienna, Austria

\footnotetext{
* Speaker.

${ }^{\dagger}$ In collaboration with Ben Allanach, Debtosh Chowdhury, Sangeun Han, Seung J. Lee, Subhadeep Mondal and Manimala Mitra.
} 


\section{Introduction}

The Large Hadron Collider (LHC) has successfully completed its first phase of run. The analyses of the data collected so far, in the search for physics beyond the standard model (BSM), has come up with null results. However, to our contentment, more than a few search channels observed by one or the other experimental group show some significant excesses. For example,

- CMS has reported a $2.8 \sigma$ excess in the eejj channel with 14 events within the $1.8 \mathrm{TeV}<$ $M_{e e j j}<2.2 \mathrm{TeV}$ bins in the context of $W_{R}$ search [1].

- Similar excesses with local significances of $2.4 \sigma$ and $2.6 \sigma$ have also been reported by CMS in the $e e j j$ and $e v j j$ channel respectively, in the context of the di-LQ search for a leptoquark mass $\sim 650 \mathrm{GeV}[2]$.

- The recent measurement of $R_{K}$ by LHCb collaboration in the low di-lepton invariant mass squared region, $1 \mathrm{GeV}^{2}<q^{2}<6 \mathrm{GeV}^{2}$, is found to be [3]

$$
R_{K}^{\mathrm{LHCb}}=\frac{B r\left(B^{+} \rightarrow K^{+} \mu^{+} \mu^{-}\right)}{B r\left(B^{+} \rightarrow K^{+} e^{+} e^{-}\right)}=0.745_{-0.074}^{+0.090} \pm 0.036
$$

This corresponds to a $2.6 \sigma$ deviation from the SM prediction, $R_{K}=1.0003_{-0.00007}^{+0.00010}$.

Though the statistics for most of these search channels are very low to make any conclusive remark, it is nevertheless instructive to relook in to these analyses a bit more carefully. A common feature of most of these excesses is that they all indicate or hint towards the violation of lepton flavor universality. Lepton flavor violation (LFV) within the SM is highly suppressed even at the loop level. Hence, any experimental hint of LFV can provide clean null test of the SM. Given its importance and the fact that various BSM physics scenarios can enhance lepton flavor violation compared to the SM prediction, it is not too early to look for the possible new physics interpretations which can give rise to such excesses. The sole purpose of such a work is to prepare oneself for high statistics data due for the next phase of LHC run.

We made an attempt to explain these seemingly uncorrelated deviations within the framework of the $\mathscr{R}$-parity violating (RPV) supersymmetry (SUSY), in particular assuming only lepton flavor violating $L Q D^{c}$-type operators in the superpotential $[4,5,6]$.

\section{Model: R-parity violating supersymmetry}

$\mathscr{R}$-parity violating SUSY provides an alternative to the canonical SUSY scenario with rich collider phenomenology. In addition, the bounds coming from the LHC data can be evaded in such scenarios due to the absence of large missing energy signature. The superpotential of the RPV SUSY can be written by extending the superpotential of the minimal supersymmetric standard model with the following terms:

$$
\mathscr{W}_{R_{p}^{\prime}}=\mu_{i}^{\prime} L_{i} H_{u}+\frac{1}{2} \lambda_{i j k} L_{i} L_{j} E_{k}^{c}+\lambda_{i j k}^{\prime} L_{i} Q_{j} D_{k}^{c}+\frac{1}{2} \lambda_{i j k}^{\prime \prime} U_{i}^{c} D_{j}^{c} D_{k}^{c},
$$


where $L_{i}, E_{i}^{c}$ denote $\mathrm{SU}(2)_{L}$ doublet and singlet superfields for leptons respectively, $Q_{i}, U_{i}^{c}$ and $D_{i}^{c}$ represent the left-handed quark doublet, right-handed up-type quark singlet and right-handed down-type quark singlet superfield respectively and $H_{u}$ is the up-type Higgs superfield that gives mass to the up-type quarks.

In the context of the present study, we have worked with $\lambda_{i j k}^{\prime}$-type RPV coupling only. In particular, we assumed non-zero value of $\lambda_{112}^{\prime}$ and $\lambda_{113}^{\prime}$ couplings, setting all other $\lambda^{\prime}$ couplings ${ }^{1}$ to zero in order to explain the experimental data. The choice of these parameters are already constrained by the existing low energy data. The region of parameter space which successfully explain the anomalies of CMS and LHCb is found to be consistent with existing experimental constraints.

\section{Results}

We have consider resonant slepton production of first generation at the LHC followed by its decay to $e e j j$ (and $e v j j$ ) final state

$$
\begin{gathered}
p p \rightarrow \tilde{e}_{L} \rightarrow e \chi_{1}^{0} \rightarrow e e j j \\
p p \rightarrow \tilde{v}_{L} \rightarrow e \chi_{1}^{+} \rightarrow e e j j
\end{gathered}
$$

via the combination of $\mathscr{R}$-parity conserving and violating interactions as described in figure 1 . The corresponding decay branching ratios are plotted in figure $2 \mathrm{a}$ for four different cases representing the gaugino admixtures of the lightest SUSY particle.
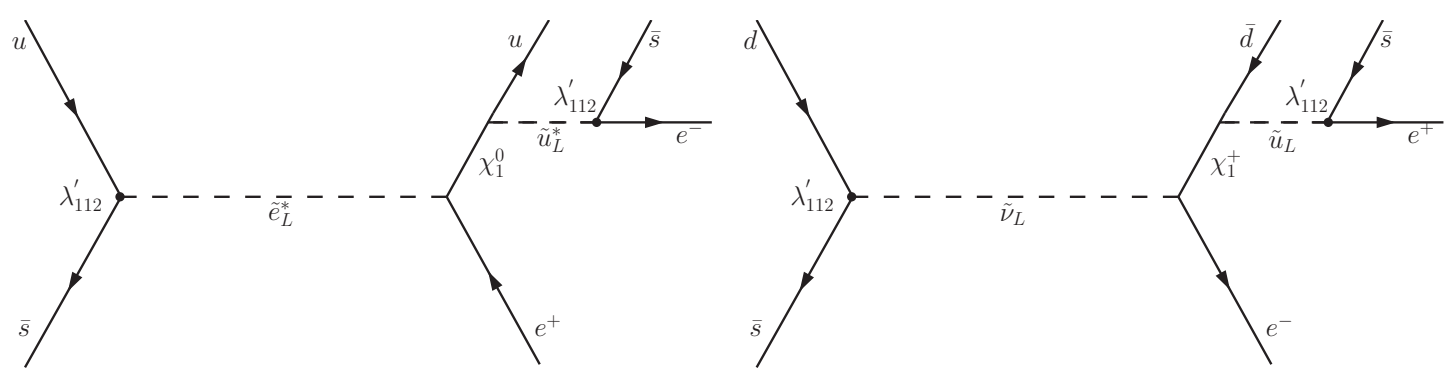

Figure 1: Resonant slepton production leading to $e e j j$ final-state at the LHC.

After applying the set of cuts described in [1] the corresponding invariant mass distribution of the $e e j j$ pair can be found in figure $2 \mathrm{~b}$ for a representative benchmark point assuming a slepton mass of $2 \mathrm{TeV}$.

The $\mathrm{LHCb}$ results can be interpreted as an enhancement in the $\mathrm{BR}\left(B^{+} \rightarrow K^{+} e^{+} e^{-}\right)$due to the presence of the following term in the lagrangian

$$
\mathscr{L} \ni \lambda_{112}^{\prime} \tilde{u}_{L}\left(\bar{s} P_{L} e\right)+\lambda_{113}^{\prime} \tilde{u}_{L}\left(\bar{b} P_{L} e\right)+\text { h.c. } .
$$

The corresponding Feynman diagram of $\bar{b} \rightarrow \bar{s} e e$ transition and allowed parameter space for LHCb observation are given in figure $3 \mathrm{a}$ and $3 \mathrm{~b}$, respectively.

\footnotetext{
$1_{\text {to explain CMS } W_{R} \text { excess one needs either }} \lambda_{111}^{\prime}$ or $\lambda_{112}^{\prime}$ coupling, however, only the combination of $\lambda_{112}^{\prime}$ and $\lambda_{113}^{\prime}$ can explain both CMS and $\mathrm{LHCb}$ results.
} 

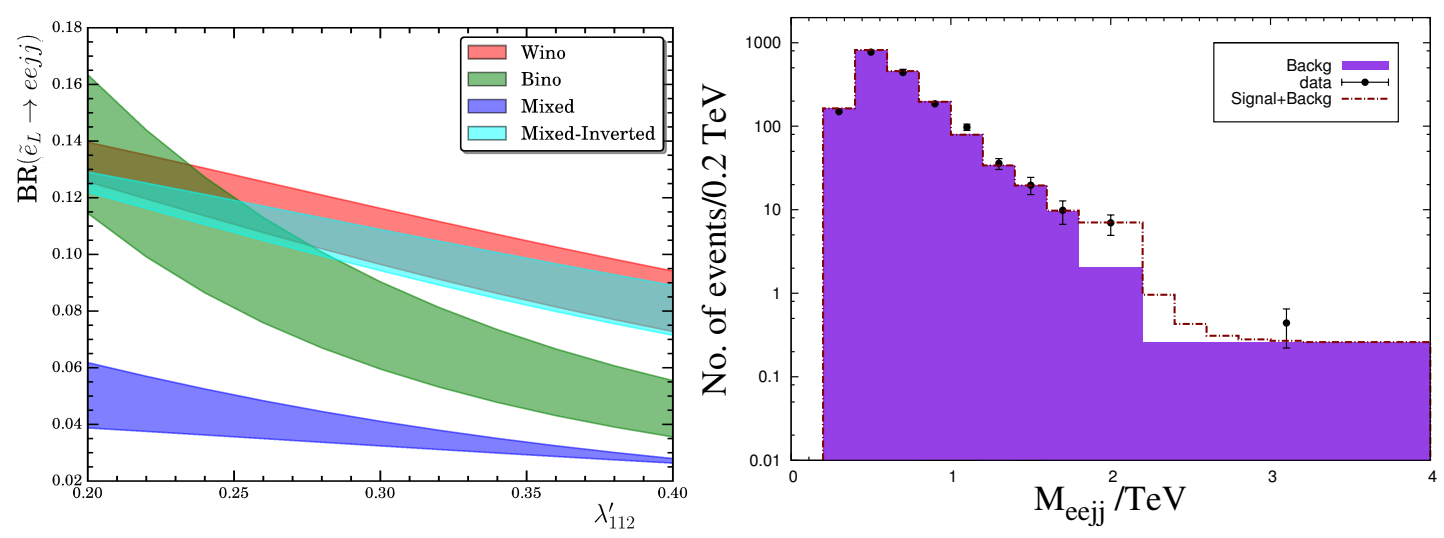

Figure 2: (Left) The effective branching ratio of the decay $\tilde{\ell} \rightarrow e e j j$ vs $\lambda_{112}^{\prime}$ and (right) A comparison of the data, signal and background $M_{e e j j}$ distributions after imposing cuts as done in the analysis of the $W_{R}$ search. The slepton mass is assumed to be $2 \mathrm{TeV}$.
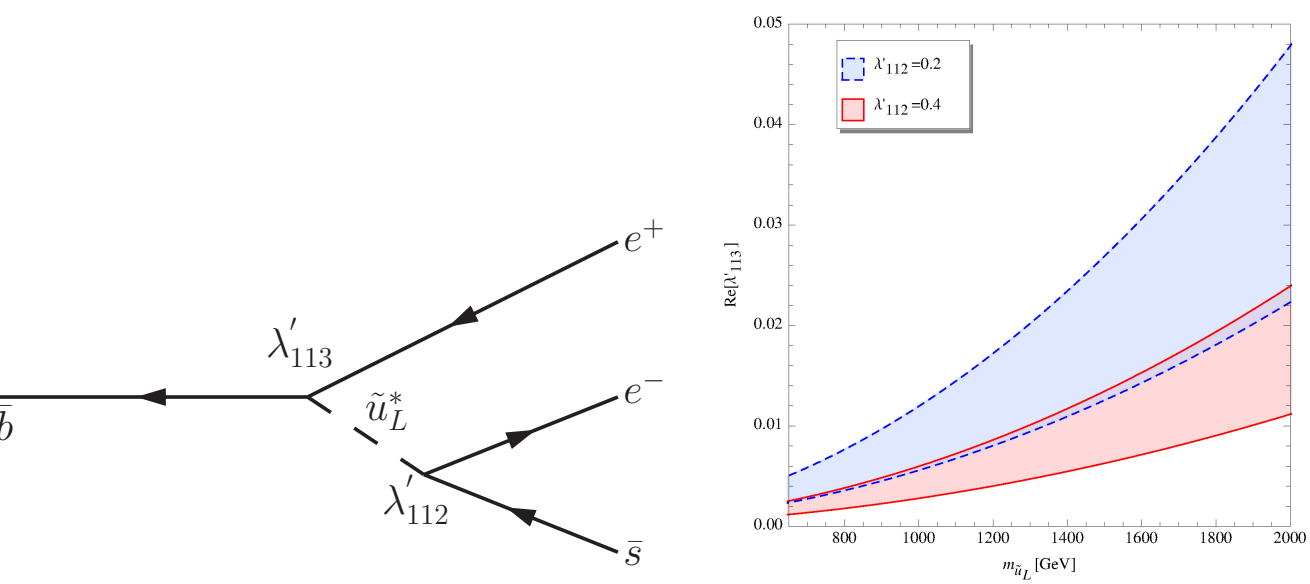

Figure 3: (Left) The Feynman diagram of $\bar{b} \rightarrow \bar{s} e e$ decay and (right) the allowed parameter space in the $m_{\tilde{u}_{L}}-\operatorname{Re}\left[\lambda_{113}^{\prime}\right]$ plane consistent with the measurement of $R_{K}$ at LHCb and other $B$-physics observables. The blue (red) colored region corresponds to $\lambda_{112}^{\prime}=0.2(0.4)$.

Figure 4 explains the allowed region of parameter space consistent with all of these observations including the dijet bound coming from the LHC data. It also shows the region of interest related to the neutrinoless double beta decay.

\section{Conclusions}

We have tried to explain the apparently small deviations observed in the CMS and the LHCb results within a unified framework of the RPV SUSY. We have shown that the flavor violation introduced by the $\lambda_{112}^{\prime}$ and $\lambda_{113}^{\prime}$ couplings can consistently explain the observed discrepancies within the experimental uncertainties. However, one need to further probe this possibility at the future LHC or other experiments with a dedicated analysis to confirm its validity. 


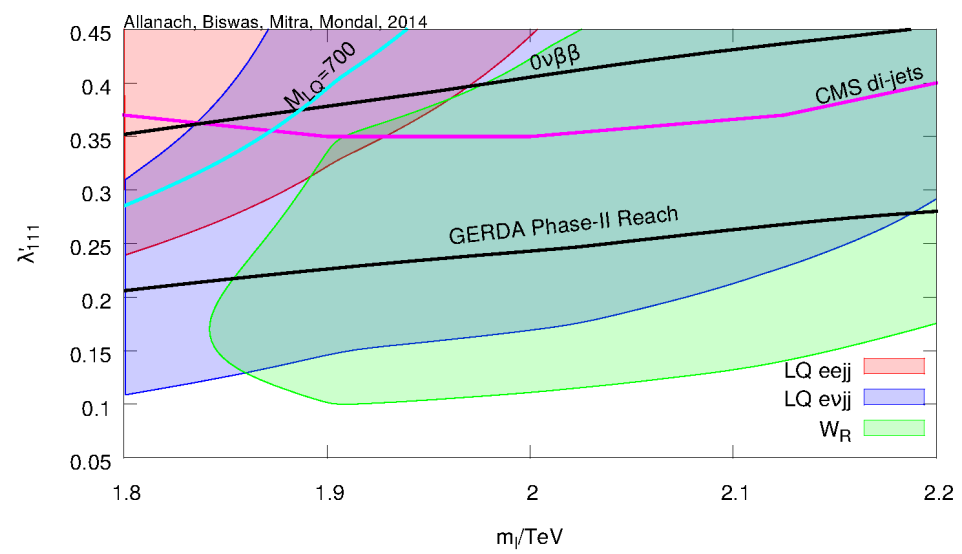

Figure 4: Compatibility between $W_{R}$ and dileptoquark searches in the $m_{\tilde{\ell}}-\lambda_{111}^{\prime}$ plane assuming $M_{\chi_{1}^{0}}=0.9$ $\mathrm{TeV}$ (similar plot can be made in the $m_{\tilde{\ell}}-\lambda_{112}^{\prime}$ plane as well).

\section{References}

[1] V. Khachatryan et al. [CMS Collaboration], Eur. Phys. J. C 74 (2014) 11, 3149 [arXiv:1407.3683 [hep-ex]].

[2] CMS Collaboration [CMS Collaboration], CMS-PAS-EXO-12-041, https://cds.cern.ch/record/1742179

[3] R. Aaij et al. [LHCb Collaboration], Phys. Rev. Lett. 113 (2014) 151601 [arXiv:1406.6482 [hep-ex]].

[4] B. Allanach, S. Biswas, S. Mondal and M. Mitra, Phys. Rev. D 91 (2015) 1, 011702 [arXiv:1408.5439 [hep-ph]].

[5] S. Biswas, D. Chowdhury, S. Han and S. J. Lee, JHEP 1502 (2015) 142 [arXiv:1409.0882 [hep-ph]].

[6] B. C. Allanach, S. Biswas, S. Mondal and M. Mitra, Phys. Rev. D 91 (2015) 1, 015011 [arXiv:1410.5947 [hep-ph]]. 